\title{
Article \\ Electronic Active Calibration of Fluxgate Magnetometers
}

\author{
Gustavo Cabral ${ }^{1 *}$, André Wiermann ${ }^{1}$ and Luiz C. C. Benyosef ${ }^{1}$ \\ 1 Observatorio Nacional/MCTI, Coordenacao de Geofisica, Rio de Janeiro 20921-400, Brazil; \\ gustavocabral@on.br (G.C.); andrew@on.br (A.W.); benyosef@on.br (L.C.C.B.) \\ * Correspondence: gustavo.cabral@ieee.org
}

\begin{abstract}
Fluxgate magnetometers require calibration methods appropriate to their application levels and particularities; however the development of fully controlled calibration procedures presents a particular challenge regarding the inevitable influence of the local geomagnetic field and other external interferences when a laboratory with magnetically shielded walls is not available. In that context, we discussed the development of an automated calibration method for fluxgate magnetometers, considering those limitations in time and space, and avoiding some of the problems commonly found in other proposed solutions for the same challenge. For this task, we designed and built a new set of high level procedures, electronic systems and software, which perform active testing and automated calibration of fluxgate magnetometers, considering some resource constraints and employing instruments commonly found in electronic calibration laboratories.
\end{abstract}

Keywords: fluxgate magnetometer; magnetic sensor; calibration; automated systems; geophysics; geomagnetic field

\section{Introduction}

Although the first fluxgate magnetometer appeared in the early 1930s, these sensors are still widely used in many applications and the fluxgate effect still attracts the interest of many theoretical works [23]. Traditionally, fluxgate magnetometers measure continuous or alternating low-frequency magnetic fields and they are intrinsically vector magnetometers, being sensitive to field direction. This type of sensor can typically achieve resolutions up to $10 \mathrm{pT}$ and allow measurements up to $1 \mathrm{mT}$. [27].

Fluxgate magnetometers require the use of specific calibration and test techniques in order to obtain their calibration coefficients and to confirm their technical characteristics. The calibration coefficients can be obtained through different methods, among them, the inference by experimental method through the active and controlled variation of the physical parameters that result in the corresponding variations of the measurements performed by the magnetometer under test. Those coefficients correlate, in the case of triaxial magnetometers, the intensity, direction and amplitude of the magnetic field to which the instrument is subjected, with its response signals.

This work shares ideas from other fluxgate magnetometer calibration systems, like [28], [29], [30], [31], [32] and [33], but addresses a different approach to avoid some of the main issues found in the solutions adopted in those other works. Our developed solution attempts to avoid some of the complexities and problems commonly associated with the use of moving parts and the need for a laboratory with magnetically shielded walls. Systems using triaxial rotation of the magnetometer with respect to a fixed reference magnetic field require the development of special non-magnetic rotation systems, and the use of moving parts imposes additional adjustments to be made due to mechanical inaccuracies and tendency of natural degradation caused by its mechanical wear with the continued use. The need for magnetic shielded environments requires the construction of special rooms of relatively high cost. 
Therefore, as a different solution, in this work were developed a set of procedures, electronic systems and software tools that allow the active testing and calibration of fluxgate magnetometers, by electronically imposing the variation of the physical parameters to which they are subjected and the measurement of their signals, so that it overcomes some of the following key limitations and challenges:

1. Asymmetry and non-homogeneity of the local magnetic field, in addition to possible electromagnetic interference;

2. Use of instrumentation typically found in laboratories for fluxgate magnetometer development; 3. Development of original computer programs that allow the automatic control of electronic instruments for controlled variation of the test parameters and acquisition of the response signals of the magnetometers under test;

4. Integration of precision electronic instrumentation with the automatic control and data acquisition system;

5. Development of original computer programs and support infrastructure for long-term comparative tests.

Figure 1 and 2 show, respectively, the diagrams of the parts that comprises our system for the exclusively magnetic characterization of the fluxgate magnetometers, which can be of analog or digital output, and the system that includes the thermal characterization of the magnetometers, since it is expected that the temperature variation has an important influence on the response curve of the instrument.

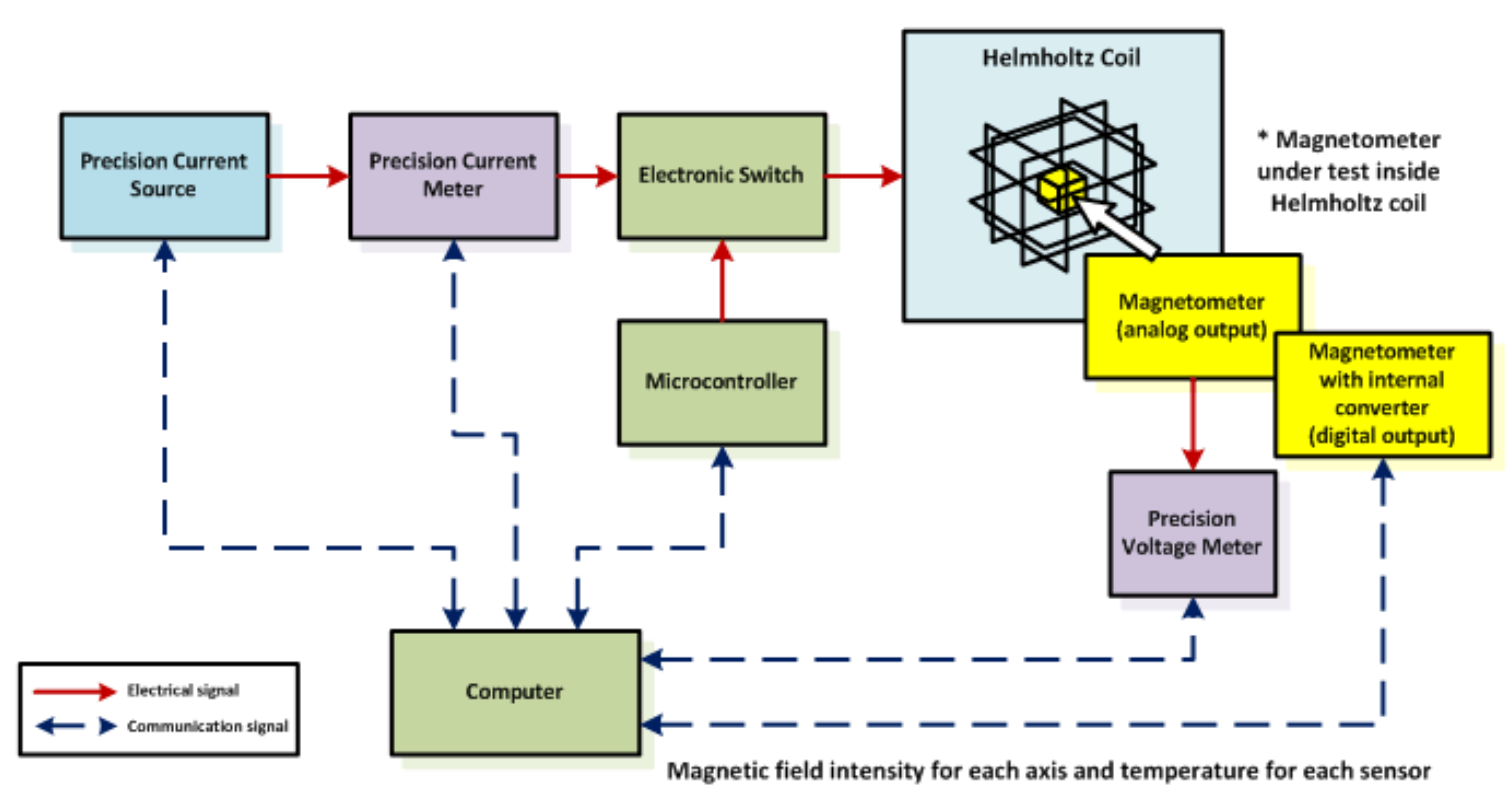

Figure 1. Magnetic calibration system for magnetometers with analog or digital outputs 


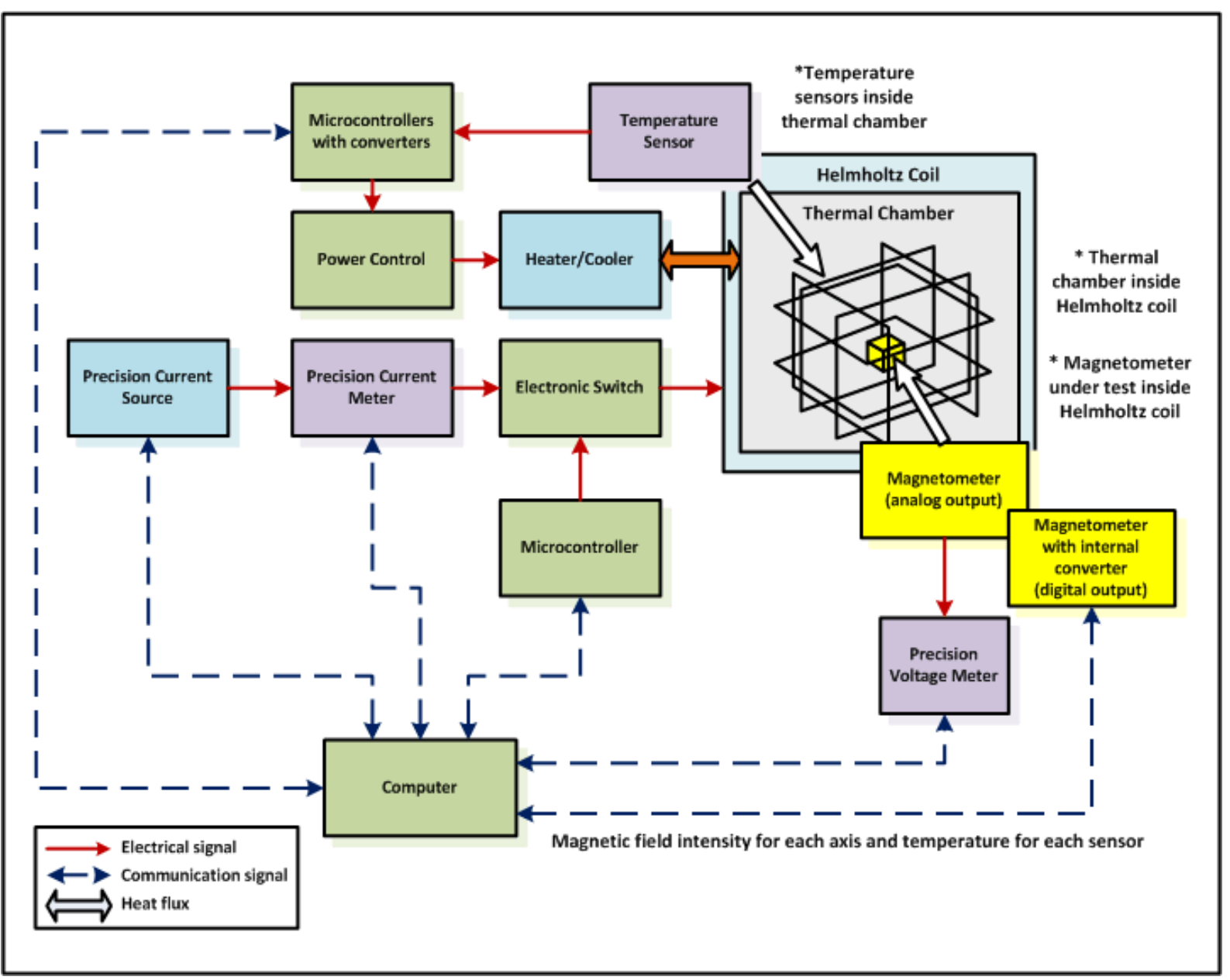

Figure 2. Magnetic calibration system including thermal characterization

\section{Materials and Methods}

A number of limitations prevents the existence of ideal conditions for the calibration of fluxgate magnetometers, therefore were selected and developed in this work a set of tools and processes that aim to minimize those limitations inherent in the nature of sensors, instruments, surrounding environment and test accessories. With this, it was possible to construct a calibration system even under strict conditions, but meeting the quality requirements for calibration of precision magnetometers.

\subsection{Proposed Method}

In simple terms, the active calibration method used in this work can be described as a method using a magnetic field artificially generated, under controlled conditions and known temperature, for which the magnetometer under test is subjected so that its response curve can be determined. However, as mentioned above, several factors make it unfeasible to generate an ideal reference magnetic field.

Since there is no materialized magnetic standard, it is necessary to provide electric means of generating the magnetic field of reference to be used in the calibration. In this work, the reference magnetic field used to excite the instrument under test is created by applying controlled electric current in pairs of solenoidal coils in parallel, forming a Helmholtz coil arrangement, where the reference magnetic field has direction given by the arrangement of the coils and polarity of the applied electric current.

Having generated a reference magnetic field, it could be proposed the simple comparison of the magnetometer in test with another magnetometer taken as standard and submitted to the same magnetic field. However, the magnetic field is influenced by everything it pervades, and especially by 


\section{the magnetic core of the sensor that has high magnetic permeability. In this way, the mere presence of another magnetometer in the vicinity of the instrument under test would be sufficient to distort the magnetic field created as a reference and invalidate the calibration. \\ Since it is not possible to use a second side-by-side magnetometer as a single calibration reference, the field generated by the external coils becomes the reference to be used and its value must be precisely known. However, the generated magnetic field will always be added vectorially to the local magnetic field, so any measurement will actually be the measurement of the resultant of these fields and not only of the generated magnetic field. \\ In summary, the method proposed in this work aims to overcome some of the difficulties found in the calibration of fluxgate magnetometers such as:}

1. Influence of unpredictable variations of the geomagnetic field in time and space;

2. Impossibility of simple comparison with the use of a second reference magnetometer side by side;

3. Absence of a materialized magnetic pattern;

4. Difficulty in obtaining a zero amplitude magnetic field;

5. Interactions of the magnetic field with the surrounding environment and consequently also with the measuring means.

In order to minimize the influence of the variation of this local magnetic field, both in time and space, and the interaction of the magnetic field with the environment and consequently with the measuring instruments, two main steps are performed:

In the first step, a high-precision magnetometer with calibration certificate is taken as the first reference standard and subjected to a long-term sequence of multiple measurements of magnetic fields of different amplitudes generated by the Helmholtz coil. Thus, the parameters that define the characterization of the Helmholtz coils are obtained. In such a way that a certified standard instrument is used to recalibrate the Helmholtz coil under the local conditions, considering the influence of its surroundings on the distortions of the local magnetic field added to the field generated by the coils, as well as the temperature at which the system is submitted.

In the following step, the standard magnetometer is replaced by the magnetometer to be tested, being precisely placed in the same position and alignment of the previous reference magnetometer. The magnetometer to be characterized is then excited by applying a sequence of magnetic fields of reference along a range of different intensities, in order to obtain the survey of its calibration curve generated by the Helmholtz coil that was specifically adjusted in the first step for this specific environment.

For each magnetic intensity value used as reference, multiple fast cycles of magnetic reversion are applied. The reversal cycles are used so that the subtraction of maximum and minimum measurement values, given respectively by the direct and reverse measurements for each time interval, results in the subtraction of the average intensity of the local geomagnetic field that acts as an overlap. Each reversal cycle is performed fast enough to overcome the magnitude of the natural variations of the geomagnetic field.

With multiple measurements available for each reference signal, a statistical filtering algorithm using robust averaging method can be applied to minimize the effects of noise on magnetic field measurement signals. Such noises come from unavoidable and distinct sources of magnetic, electrical and thermal interference.

This method, using electrically controlled process of magnetic field reversal cycles, is equivalent to the mechanical rotation process used in other methods, but without the issues usually found in solutions that have moving parts.

\subsection{Calibration Parameters}

The calibration of a magnetometer results in the determination of parameters that describe a unique relationship between the response signals, obtained by magnetometer internal sensors through the associated electronic circuits, and the value of the reference magnetic field applied to the magnetometer under test. Such calibration parameters can also be understood as the parameters of 
the mathematical model that describes the behavior of the magnetometer as a function of its input signals. Therefore, these parameters allow the conversion of the raw output signal value, either analog or digital, into the measurement result representing the estimated magnetic field.

Based on the premise that the raw signal from the magnetometer output varies linearly with respect to the magnetic field to which it is subjected, the calibration characteristic curve can be estimated from a linear mathematical model of magnetometer behavior. That model can be described by linear regression using the least squares method. This is a widely recognized modeling method used in linear regression analysis for the approximation of measurement solutions, especially overdetermined systems that have a normal statistical distribution of noise. That adjustment is made so that the mean square error of the input data set relative to the reference values is minimized.

However, for such a premise to be valid, it is required that as many measurements as possible for the same reference value are performed. In addition, spurious values must be filtered, that is, values that are statistically very far from the expected value should be removed. Consequently, an automated computer system was developed to enable this extensive acquisition of data with more consistent results as it avoids human deviations that are normally present in manual calibrations.

It is expected that the raw output signal of any magnetometer under test will be reasonably influenced by other factors, especially temperature variations, since the measurements taken by the magnetometer are directly related to the dimensions of the elements that make up the sensor, such as excitation and sensing coils, constructed with long copper windings that present expansion and contraction with temperature variations. Other parts that may also be influenced by temperature variation are the core material and the electronic components of the amplification and data acquisition circuits. Therefore it is important to evaluate the dependence of the output signal of the magnetometer with the temperature variation. Considering that, for the temperature variation to be also taken into account in the magnetometer response modeling, a simple linear regression method is not sufficient and multiple linear regression modeling is used, where the response variable is defined as linearly dependent on two or more independent variables.

The linear regression parameters are found considering the relationship between the reference magnetic field, the raw data output of the magnetometer under test and its temperature variation as described by the following equation:

$$
B_{\text {reference }}=C_{3} \times \text { RawData } \times\left(t-t_{0}\right)+C_{2} \times \text { RawData }+C_{1} \times(t-t 0)+C_{0}
$$

Where $B_{\text {reference }}$ represents the reference magnetic field applied by the Helmholtz coils, considering already a sequence of inversions on the applied magnetic field to subtract the influence of local geomagnetic field; RawData is the raw output signal of the magnetometer under test, given after the analog to digital conversion provided by the magnetometer circuits or external conversion; $t_{0}$ is temperature of reference; and $C_{0}$ and $C_{3}$ are the adjustment parameters to be found through the linear regression method using a significant and satisfactory number of measurements along the range of interest, in terms of magnetic field and temperature variations.

\subsection{Calibration Procedure}

The fluxgate magnetometer calibration procedures developed in this work can be grouped into two main procedures:

1. Characterization in near zero magnetic field with or without temperature variation;

2. Characterization with variable magnetic field applied with or without temperature variation;

In the first procedure, the magnetometer under test is placed inside a magnetic or thermo-magnetic shield (Figures 1 and 2) and interconnected to the calibration system for reading the measurements, filtering the measurements and estimating the offset, with the influence of the thermal variation being considered or not. 
In the second procedure, the magnetometer is positioned inside the Helmholtz coil, which may also include thermal chamber for the corresponding thermal characterization. In this group, the calibration program is used to apply different magnetic field values on the magnetometer under test through the Helmholtz coil and associated instruments.

This variation of the magnetic field allows the determination of the other calibration coefficients by means of the correlation between the values applied and the values measured after several cycles of variation of the applied field, filtering of the measured values and compensation of the influence of the local field.

\section{Instrumentation and Facilities}

This section describes the main tools, precision instruments and accessories selected or especially developed for the systems in this work.

\subsection{Helmholtz Coil}

The Helmholtz coils is one of the main tools for magnetometer calibration. The coils are constructed in pairs of identical solenoid coils driven by the same electric current and assembled symmetrically on the same axis and separated by such a distance as to produce an approximately uniform magnetic field at the equidistant central point. They are named after the physicist Hermann von Helmholtz.

The magnetic field that is generated by a coil of a given geometry depends on the position relative to the coil. Assuming that this coil system is large enough, there will be a central volume in which the field is homogeneous to some level. In general, this volume is small compared to the size of the coil. In theory, an infinite-length solenoid will produce a homogeneous magnetic field within its limits $[10,16]$.

To allow the generation of magnetic fields in all directions, a set of three pairs of orthogonal coils with a common center is used. In this way, the combination of amplitudes of the coils of each axis determines the direction and the intensity of the resulting magnetic field. The coils used in this project have a square section as can be seen in Figure 3.

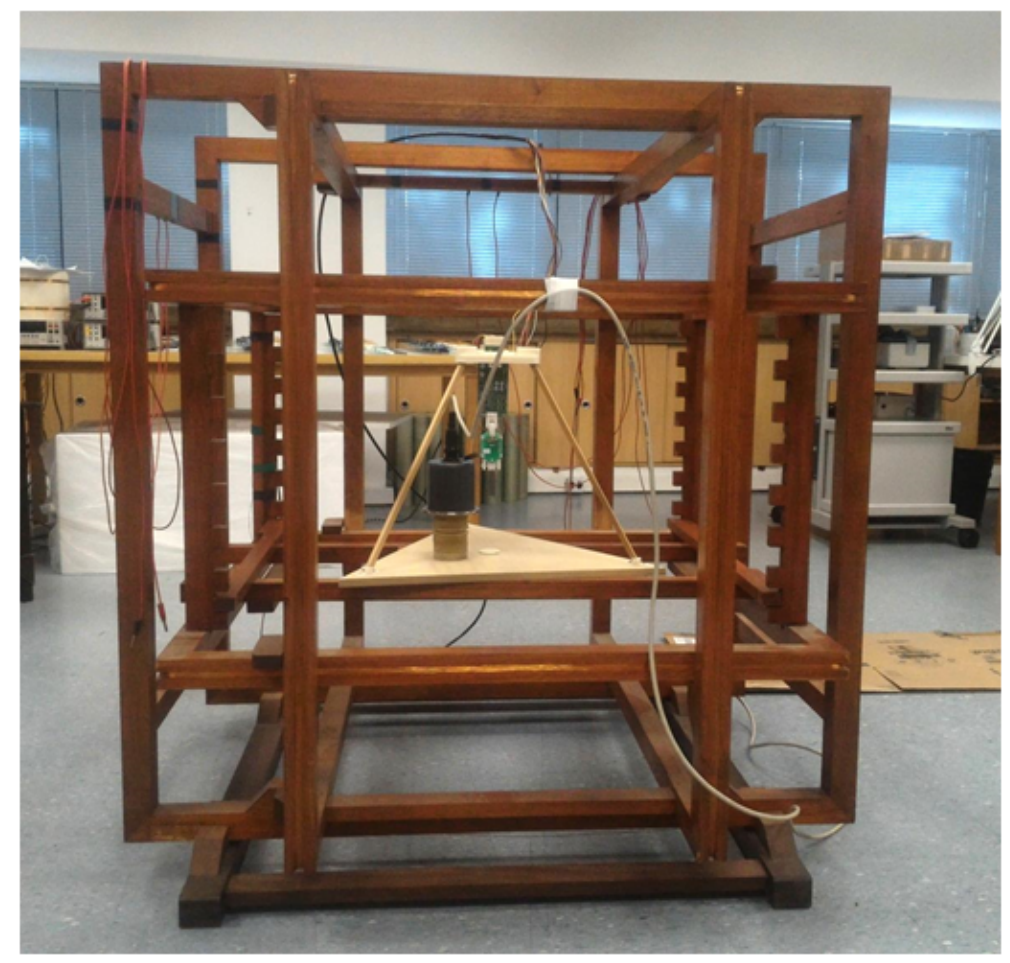

Figure 3. Image of the triaxial arrangement of Helmholtz coils used in this project 
An important limitation to be overcome in this work is the absence of a magnetically protected room to shield the Helmholtz coil. In this way, all the tests performed with the coil will also be influenced by the variations of the local geomagnetic field during the measurements. According to Koike et al. (1990) [13], the reference signal and the natural variations of the geomagnetic field will be superimposed, causing interference with the process in which the magnetometer produces a sensitivity value. If this interfering effect is strong, the degree of accuracy of the calibration will drop. When making measurements with geomagnetic variations in a calm state and using mean values obtained through repeated measurements, it is possible to achieve $0.1 \%$ accuracy.

\subsection{Magnetic Shield}

One of the important parameters in the magnetometer calibration process is the determination of its response to a zero magnetic field, which in practice translates into a minimum magnetic field. The determination of this parameter is termed as offset characterization or characterization of response to the minimum magnetic field. As the magnetic flux from the geomagnetic field is present everywhere and pervades everything it encounters, obtaining a zero intensity magnetic field is impractical. As the magnetic flux can not be contained, one of the practical solutions used is the deviation of the flow through a called magnetic shield. This shield consists of the use of sheets of a material of high magnetic permeability arranged in a suitable mechanical arrangement in order to deflect to the maximum the magnetic flux, represented by its field lines, into the interior of this material and thus creating a volume in the space outside with minimal magnetic flux. This type of shielding by using only passive elements are here defined as passive magnetic shielding.

Materials used in passive shielding typically have very high relative magnetic permeability $(\mu / \mu 0>10000)$. Some examples of metal alloys used in the shield are Mu-metal (composed of approximately $80 \%$ nickel, $5 \%$ molybdenum and small amounts of various other elements) and Permalloy (composed of approximately $80 \%$ nickel and $20 \%$ of iron content). In practice, several concentric layers of encapsulation with same high magnetic permeability material are used to amplify the required magnetic flux deviation effect.

The magnetic shield used in this work is a passive magnetic container composed of five concentric Mu-metal cylinders with end caps having central openings to allow the passage of the electric cables for interconnection of the magnetometer under test. This shield is part of the LDSM-ON facilities and it was purchased before this project. Figure 4 shows this magnetic shield.

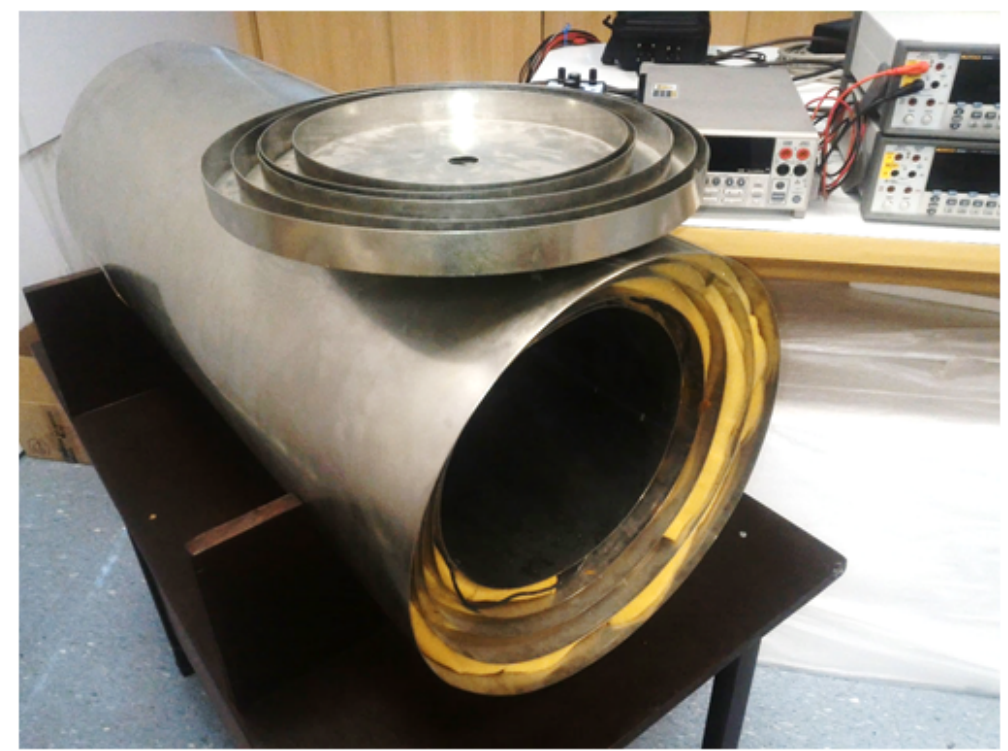

Figure 4. Magnetic shield used for near zero magnetic field characterization 


\subsection{Power Supply}

During calibration, the power supply of the magnetometer under test is carried out by a bench power supply with continuous voltage output, characterized by being sufficiently stable to avoid additional errors in the test system. That power supply has isolated programmable electrical voltage output between $0-30 \mathrm{~V}$, with maximum electric current of $3 \mathrm{~A}$ and noise level less than $1 \mathrm{~m} V_{r m s}$.

\subsection{Programmable Current Source}

The electrical signal used to produce the magnetic field in the Helmholtz Coil is produced by a programmable current source. This source is controlled by the calibration program installed on the computer to which it is connected, allowing the magnetic intensity applied to the magnetometer under test to be automatically change throughout the calibration cycles. The current source used has a resolution of $50 \mu \mathrm{A}$ for the $1 \mathrm{~A}$ operating range.

\subsection{Programmable Precision Ammeter}

Due to possible discrepancies between the value of electric current programmed to be generated by the continuous electric current source and the value effectively applied in the calibration coils, a precision ammeter is used in series with the circuit of this source. As well as the source of continuous electric current, this ammeter is also programmable and is interconnected to the computer of the calibration program. This interconnection allows the programming of the measuring scale and automatic acquisition of electrical current measurements in synchronization with the calibration cycles. The measurement through this ammeter is used to calculate the corresponding magnetic field applied to the magnetometer under test. The coefficients that correlate the magnetic field generated by the Helmholtz coils from the applied electric current are theoretically determined and adjusted based in measurements performed with a calibrated reference magnetometer.

\subsection{Programmable Precision Multimeter}

For reading the output signal of magnetometers that do not have a digital output, a precision multimeter connected to the computer of the calibration program is used. This interconnection allows the programming of the type of measurement to be performed for reading the magnetometer's analogue output, in general continuous electrical voltage signal $(0-10 \mathrm{~V})$ or continuous electric current signal (4-20 mA). In addition to selecting the type of measurement, the interconnection with the computer allows programming of the measurement scale and automatic acquisition of these measurements in synchrony with the calibration cycles as is done for the electric current signal applied to the Helmholtz coils. For the calibration of the analogue output magnetometers, consideration should be given to the conversion of the output signal value of the magnetometer, voltage or electric current, to the corresponding measured magnetic field value. The magnetometers used for the tests performed in this work have serial digital output with specific communication protocols.

\subsection{Programmable Relay Box}

The programmable relay box (Figure 5 ) has been specially designed and assembled for this work and it is responsible for receiving and translating commands from the computer via serial communication, to then switch the interconnection between the electric current source, multimeter and the Helmholtz coil. In this way, it allows to connect, disconnect or toggle the polarity of the electrical current signal applied in the coils. This device consists of a microcontrolled board connected to a relay board that is connected to the electrical current cables. 


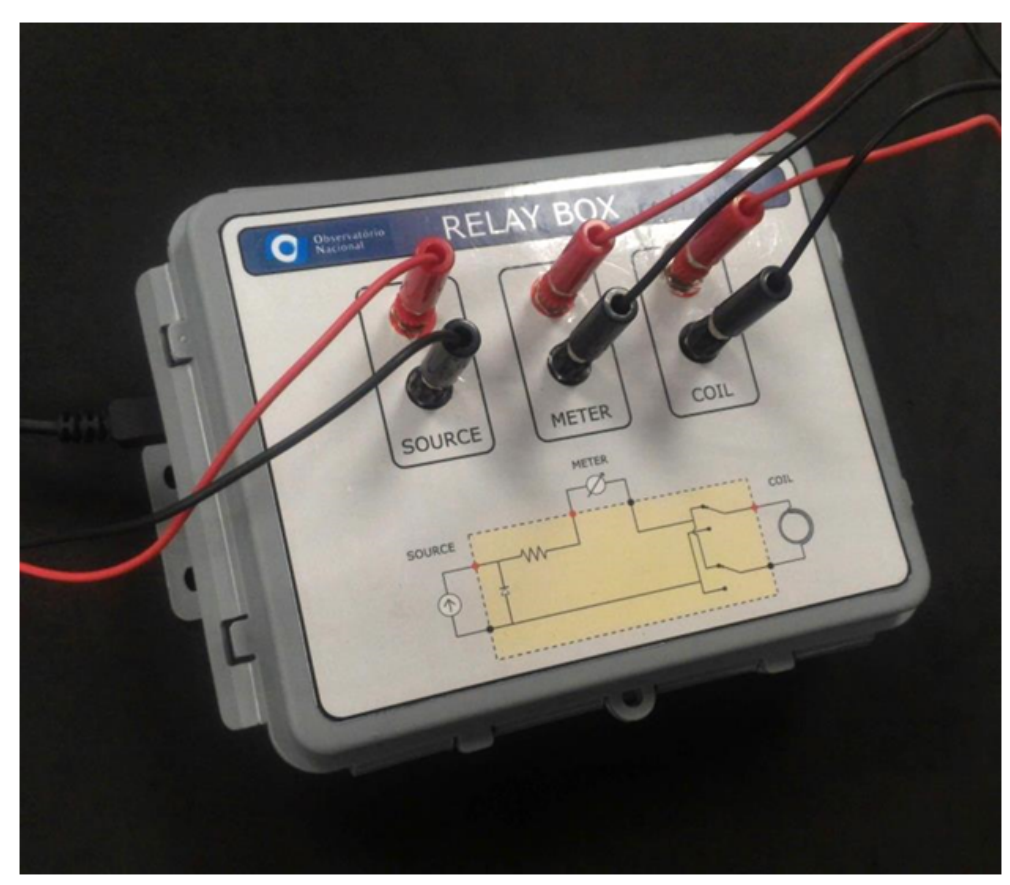

Figure 5. Programmable relay box

\subsection{Interconnection of Instruments with the Computer}

All programmable precision instruments, programmable relay box and magnetometer under test are interconnected to the calibration computer via serial communication channels. For each of those peripherals there is a unique two-way serial communication channel on the computer. Any serial communication typically imposes restrictions on transmission speed but it does not represent a significant impact considering the communication rates required by the procedure used in this work and the maximum communication speed available on both the precision instruments and the magnetometers being tested.

\subsection{Communication Protocols}

Specific serial communication protocols are used to transmit and to receive commands and measurement data between precision instruments, relay box and magnetometer under test . For the precision instruments, the communication protocol known as GPIB was used, which is specified by the ANSI / IEEE standard 488.1-2003 - IEEE Standard For Higher Performance Protocol for the Standard Digital Interface for Programmable Instrumentation. The Firmata protocol was used for the commutator box. This protocol was created for the control of microcontrollers from a computer. As a principle this protocol intends to be implemented in the internal memory of any type of microcontroller architecture and it can be controlled from any type of existing computer. Magnetometers under test have their own communication protocols, therefore every new magnetometer may require modification in the program to interpret its transmitted data.

\subsection{Calibration Computer}

The calibration computer was selected and prepared to interconnect and control all required instrumentation and fluxgate magnetometer under test by means of the calibration software. That computer is a standard PC computer with a Windows operating system and with the number of serial ports available to connect all those peripherals. 


\subsection{Calibration Software}

The calibration software (Figure 6) was especially developed for this work and it is able to perform the following main activities:

1. Configuration and management of serial communication with interconnected precision instruments and magnetometer;

2. Programming of automatic variations of the reference magnetic field applied by the Helmholtz coils in multiple configurations of cycles, time, polarity and intensity, including automatic variation of the magnetic field in long period calibration cycles;

3. Adjustment and filtering of the influence of local magnetic field;

4. Estimation of calibration coefficients for the magnetometer under test;

5. Data recording of all relevant readings in the $\mathrm{X}, \mathrm{Y}$ and $\mathrm{Z}$ axes;

6. Generation of comparative graphs and tables for applied magnetic field and measured magnetic field regarding time and temperature variations.

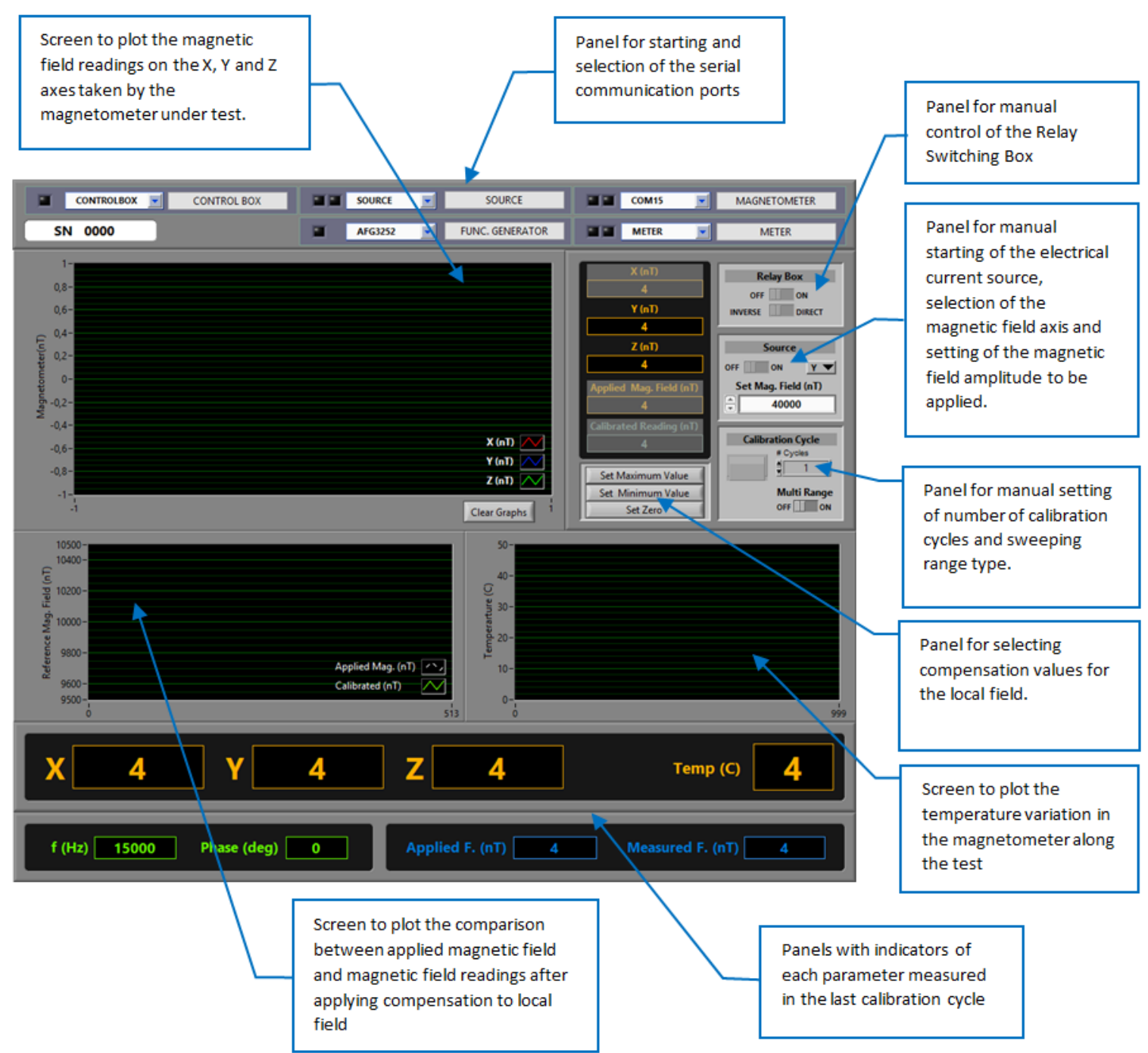

Figure 6. Picture of the main screen of the calibration program with its functions

The figure 7 presents an image of the program for interpretation and treatment of data of magnetometers developed for this work. 


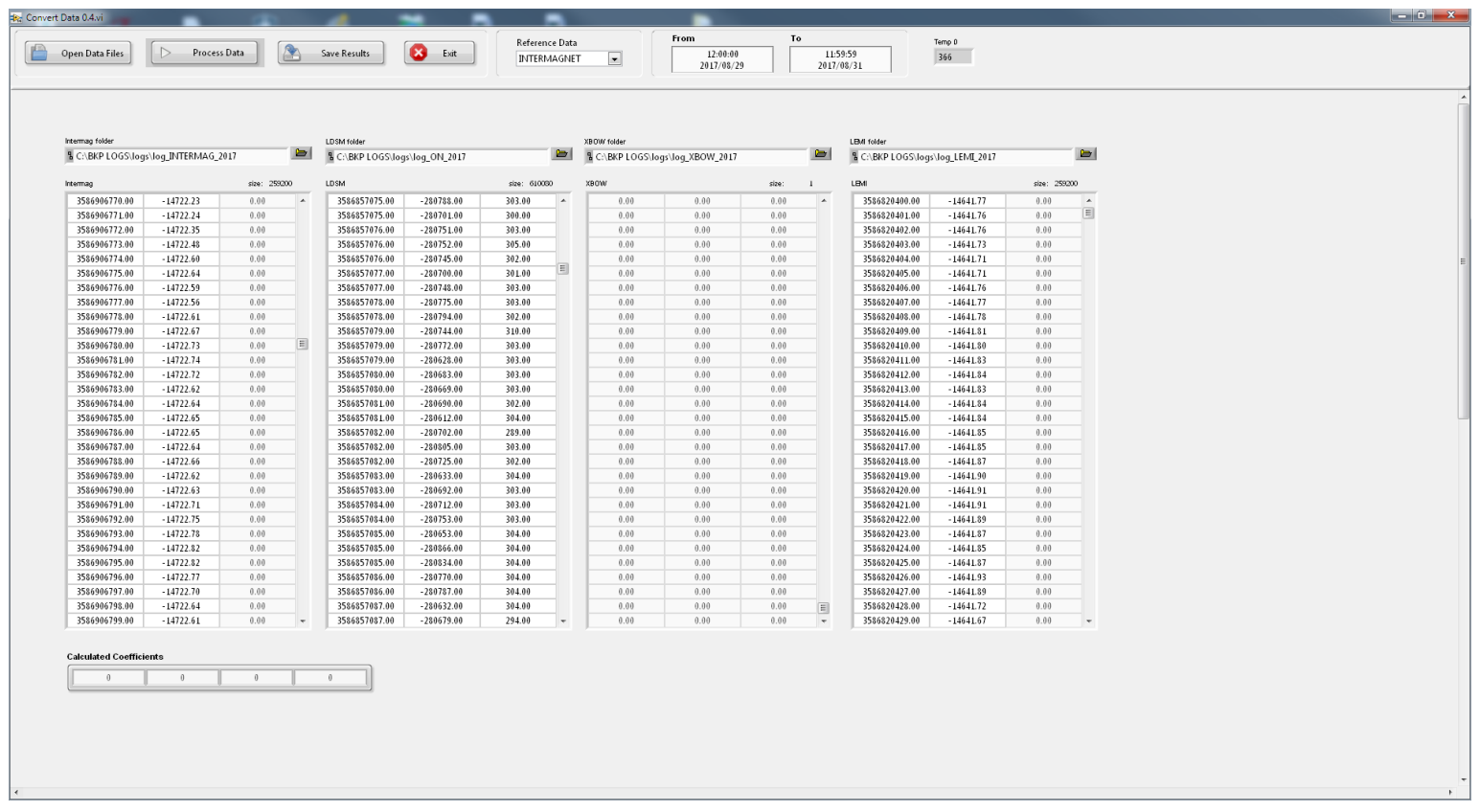

Figure 7. Magnetometer data interpretation and data processing software

\subsubsection{Computational Algorithm}

The calibration software was developed using pseudo-parallel programming with four main processes acting interleaved to control and read the precision instruments, the magnetometer under test and the automatic calibration routine. These processes can be divided into: switching control process, magnetometer measurement process, reference magnetic field measurement process, and automatic calibration process.

\subsubsection{Switching Control Process}

The switching control process manages the serial communication with the relay box using the open standard protocol called Firmata. The relay box receives the commands to turn Helmholtz coils on or off and also receives the commands for interconnecting the coils in direct or reverse polarity. Based on the received commands the internal microcontroller manages the activation of the corresponding relays.

\subsubsection{Magnetometer Measurement Process}

The process for measuring the magnetometer under test manages the communication of the computer with the magnetometer over the various calibration cycles, acquires the raw data of its output and applies the preliminary calibration coefficients for the conversion of both the magnetic field reading and its temperature. The communication with the magnetometer is made using its specific protocol, so it was adjusted for each of the different types of magnetometers used during the tests.

From the readings of the magnetometer under test, the minimum and maximum magnetic field values were recorded. The minimum magnetic field is the result of the overlapping of the applied magnetic field in the reverse direction and the local field, and the maximum magnetic field is resulting from superposition of the magnetic field applied in the direct direction and the local field. In order to find the value that corresponds only to the field applied, without the contribution of the local field, the value to be used was estimated from the multiple measurements of the mean value between the maximum and minimum magnetic field readings. 


\subsubsection{Applied Magnetic Field Measurement Process}

The magnetic field measurement process manages the communication of the computer with the precision ammeter. This process automatically sets up the metering and filtering parameters of the ammeter, and reads the electric current applied by the programmable current source to the Helmholtz coil along the calibration cycles.

This process establishes the reading of the electric current measurement according to the selected axis (X, Y or Z) and applies the correlation constant between the applied current (in $\mathrm{mA}$ ) and the corresponding magnetic field (in nT). At the end of the measurement this value is recorded as the Applied Magnetic Field.

\subsubsection{Automatic Calibration Process}

The automatic calibration process performs the control of the calibration cycles allowing configuration of the operating modes with selection of the axis to be measured and the sweeping mode in terms of magnetic field reference, including the determination of the operating range, number of calibration cycles, and multiple combinations of sweeping.

During calibration, this process is responsible for managing the communication with the programmable current source used to control the electric current applied to the Helmholtz coil and managing the communication with the other processes to control the switching sequence, the measurement of magnetic field of reference and the readings from the magnetometer under test. At the end of each measurement cycle, this process acquires the result of each of the other processes and updates the corresponding graphs and tables.

\section{Results}

\subsection{Characterization Tests for Minimum Magnetic Field}

The characterization test for minimum magnetic field, or offset characterization, was performed with the magnetometer inserted in a multilayer magnetic shield, according to the method described in section cite instruments, to determine its response to a minimum intensity magnetic field, including its response for temperature variation. A total of 20300 measurements were performed for each of the three axes with a temperature variation in the range of $22 \mathrm{C}$ to $30 \mathrm{C}$.

\subsection{Characterization Tests for Variable Magnetic Field}

The characterization tests for variable magnetic field were performed with the magnetometer at the internal central position of the Helmholtz Coil and with its axes aligned with the reference axes of the instrument. The automatic range variation cycles were configured to provide six magnetic field reference values by means of the Helmholtz Coil interconnected to the calibration system: 2500, 5000, $7500,10000,12500$ and $15000 \mathrm{nT}$. The measurements are performed in ascending and descending cycles for each of the reference values, with 21 measurement cycles per reference.

\subsection{Determination of Calibration Parameters}

As described in the proposed methodology, the calibration parameters were estimated by linear regression from the multiple values found in the characterization tests for minimum magnetic field and variable magnetic field presented above.

\subsection{Long-Term Testing}

Long-term tests were performed in order to evaluate the performance of the magnetometer with the applied calibration parameters subject to natural conditions of temperature variation and geomagnetic field variation. 
The tests were carried out in the Magnetic Observatory of Vassouras and they were of comparative nature. The magnetometers used as reference were close enough to measure approximately the same total magnetic field intensity, considering a region with a low magnetic distortion gradient, and far enough apart to avoid mutual interference.

Unlike the tests performed with the Helmholtz coil, where the produced reference field is limited to a small volume in the space at its center, the geomagnetic field is widely distributed and it can be used as a reference signal for comparative tests, provided that conditions described above are satisfied.

The Magnetic Observatory of Vassouras was designed to study the Earth's magnetic field by continuously measuring the Earth's magnetic field through its magnetometers. This observatory is part of the INTERMAGNET global observatory network, which defines quality standards and guides on instrumentation and data acquisition. Therefore this observatory is an ideal place for comparative tests between magnetometers, for the quality and reliability of uninterrupted measurements.

The INTERMAGNET (International Real-time Magnetic Observatory Network) is a consortium of institutes operating ground-based magnetometers that record the geomagnetic field. An INTERMAGNET magnetic observatory class shall be able to provide at least one-minute interval magnetic field measurements performed by a set of triaxial vector magnetometer and scalar magnetometer, all with a resolution of $0.1 \mathrm{nT}$. The measurements made by the vector magnetometer are adjusted by the best available baseline (offset adjustment) provided by the scalar magnetometer. Some observatories, such as the Vassouras Magnetic Observatory, are capable of generating one-second resolution data.

In addition to comparative tests using the official INTERMAGNET measurements, comparative tests were carried out against commercial magnetometers such as a CXM-539 triaxial vector magnetometer and a LEMI-417 triaxial vector magnetometer. The magnetometers selected for these tests are purposely distinct instruments in their applications. The LEMI-417 is a magnetometer used in geomagnetic measurement applications that require precision geophysical instrumentation. On the other hand, the CXM-539 magnetometer is generally used as a navigational and attitude control instrument for land, air or sea vehicles.

The figure fig: figure100 shows the approximated location of the instruments and control room where the INTERMAGNET's data acquisition computer and computer for data acquisition and transmission of the test magnetometer data and the other benchmark magnetometers. Due to the difficulty of alignment of the magnetometers, installed in different bases, in relation to the $\mathrm{X}$ and $\mathrm{Y}$ axes, only measurements with the relation to the $\mathrm{Z}$ axis, parallel to the gravitational vector and therefore easier to align, were chosen as reference.

All comparative figures show the output of the LSDM magnetometer after the adjustment provided by the calibration parameters found through the method implemented in this work. 


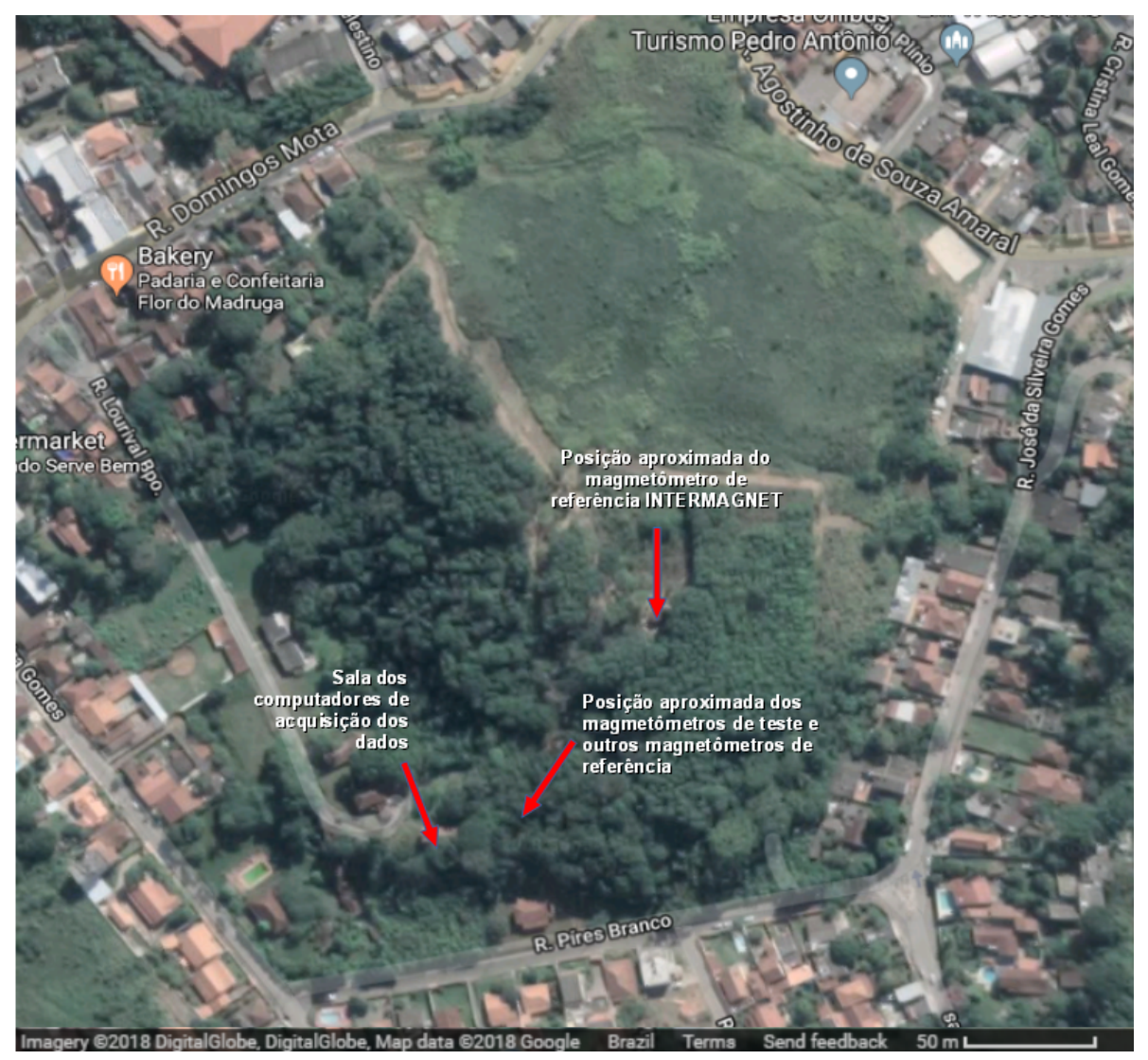

Figure 8. Relative position of instruments used in long-term measurements at the Magnetic Observatory of Vassouras

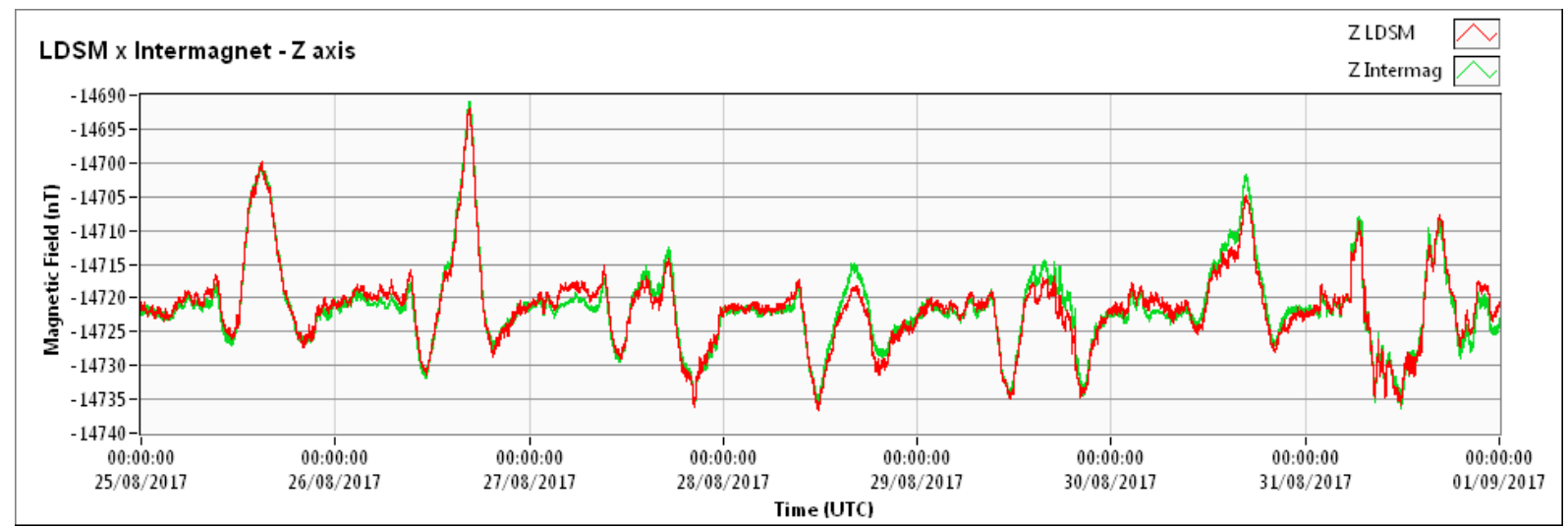

Figure 9. Example of long-term comparison between magnetometer developed in LDSM and magnetometer of reference INTERMAGNET 


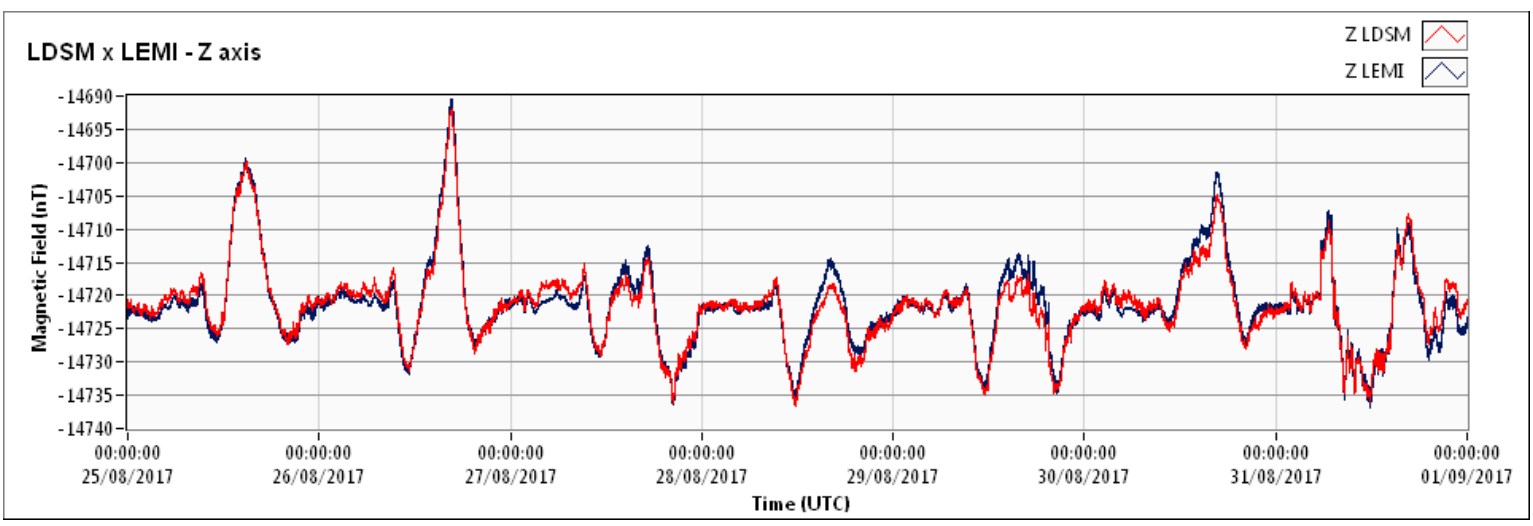

Figure 10. Example of long-term comparison between LEMI-417 commercial magnetometer and INTERMAGNET reference magnetometer

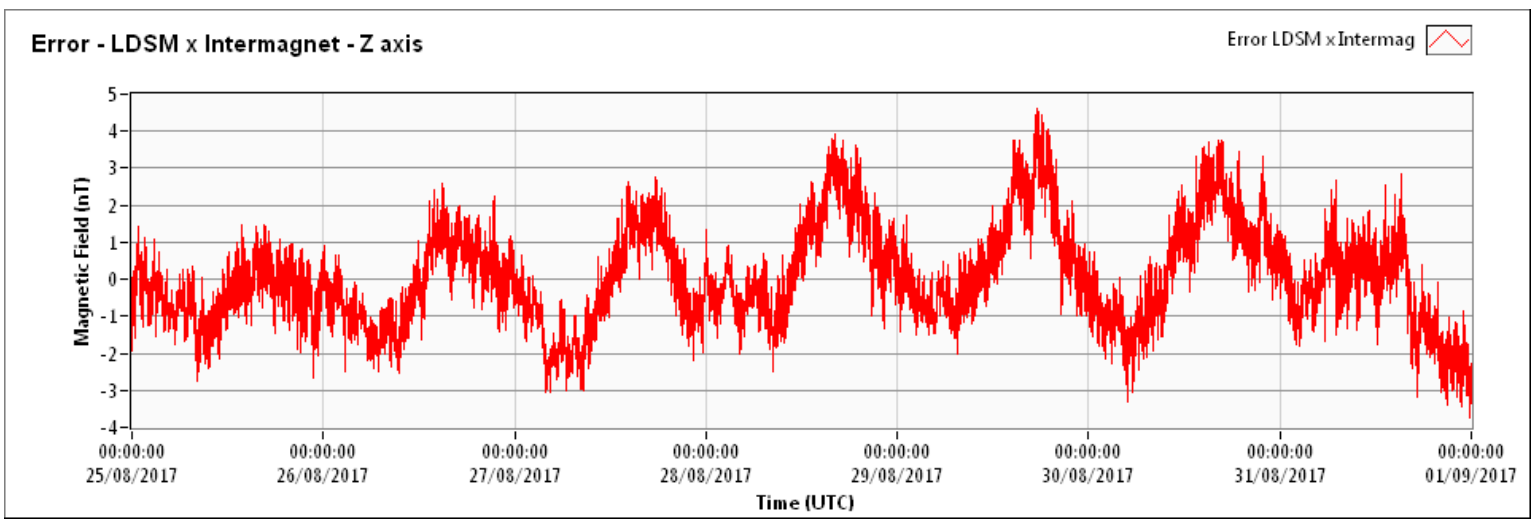

Figure 11. Example of measurement difference between magnetometer developed in LDSM and magnetometer of reference INTERMAGNET

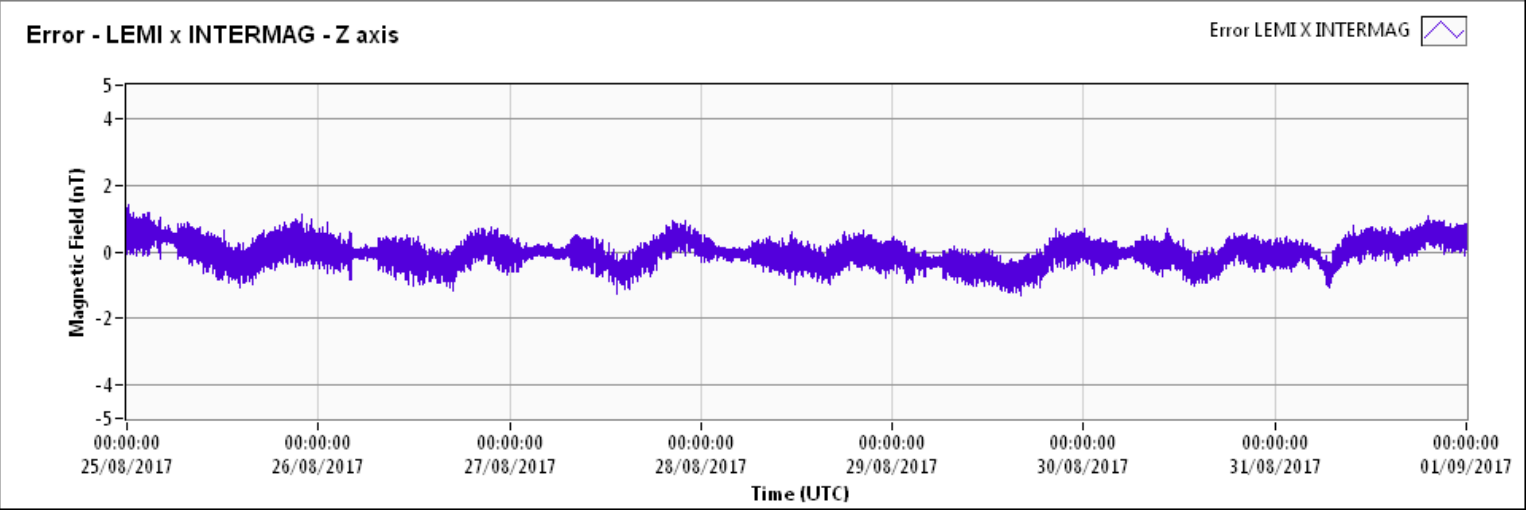

Figure 12. Example of measurement difference between LEMI-417 commercial magnetometer and INTERMAGNET reference magnetometer 


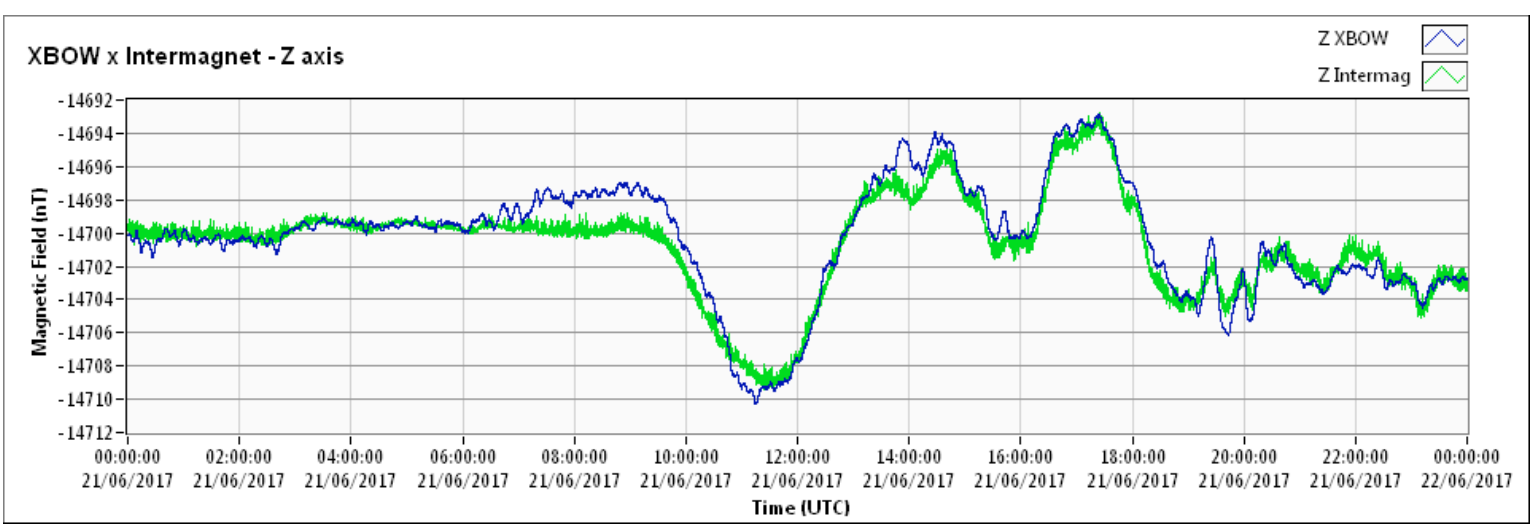

Figure 13. Example of measurement difference between CrossBow CXM539 commercial magnetometer and INTERMAGNET reference magnetometer

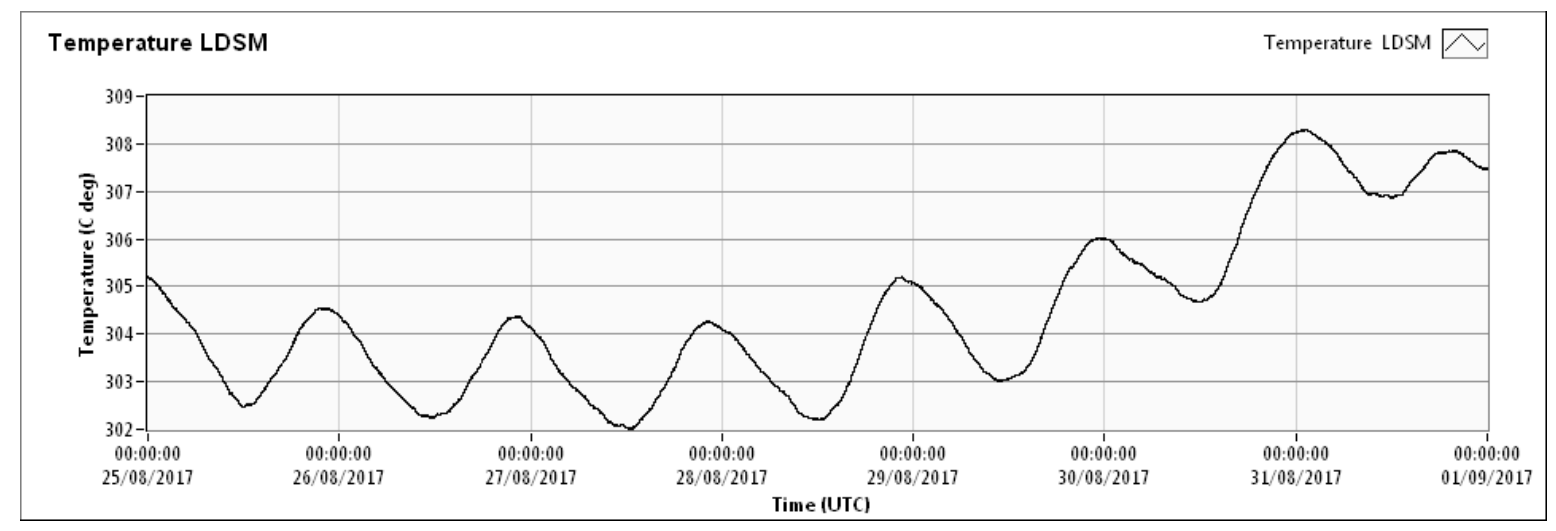

Figure 14. Temperature variation measured by magnetometer developed by LDSM (Gross Values) during long-term test

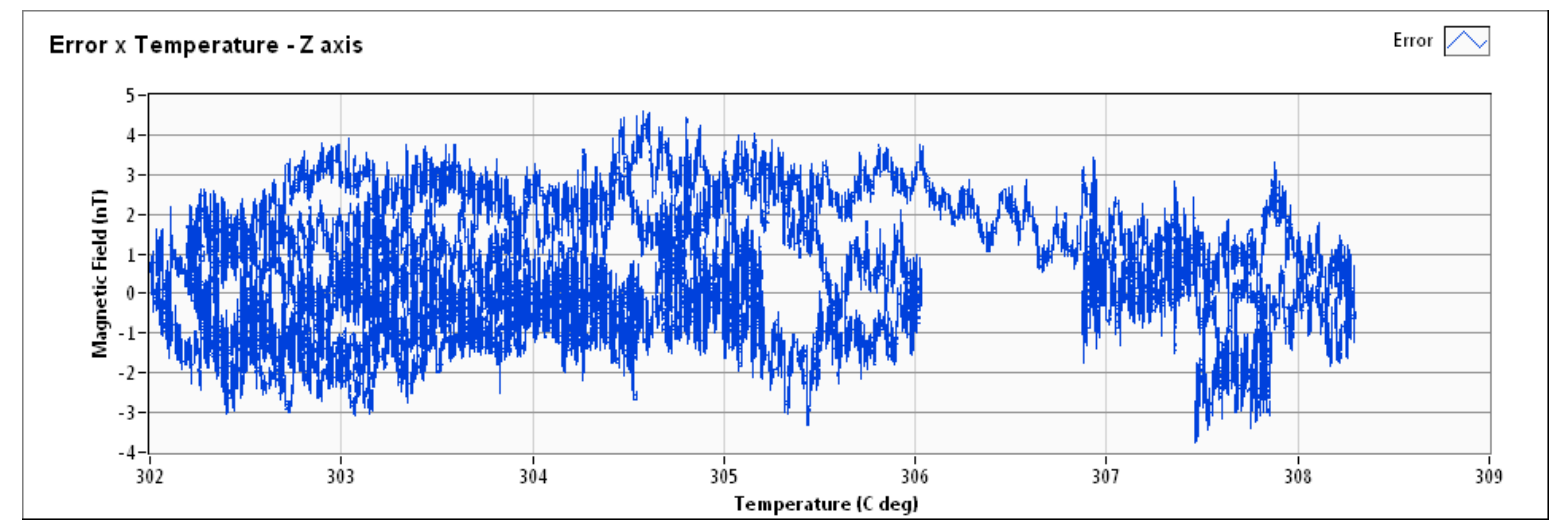

Figure 15. Variation of measurement error compared to temperature variations 


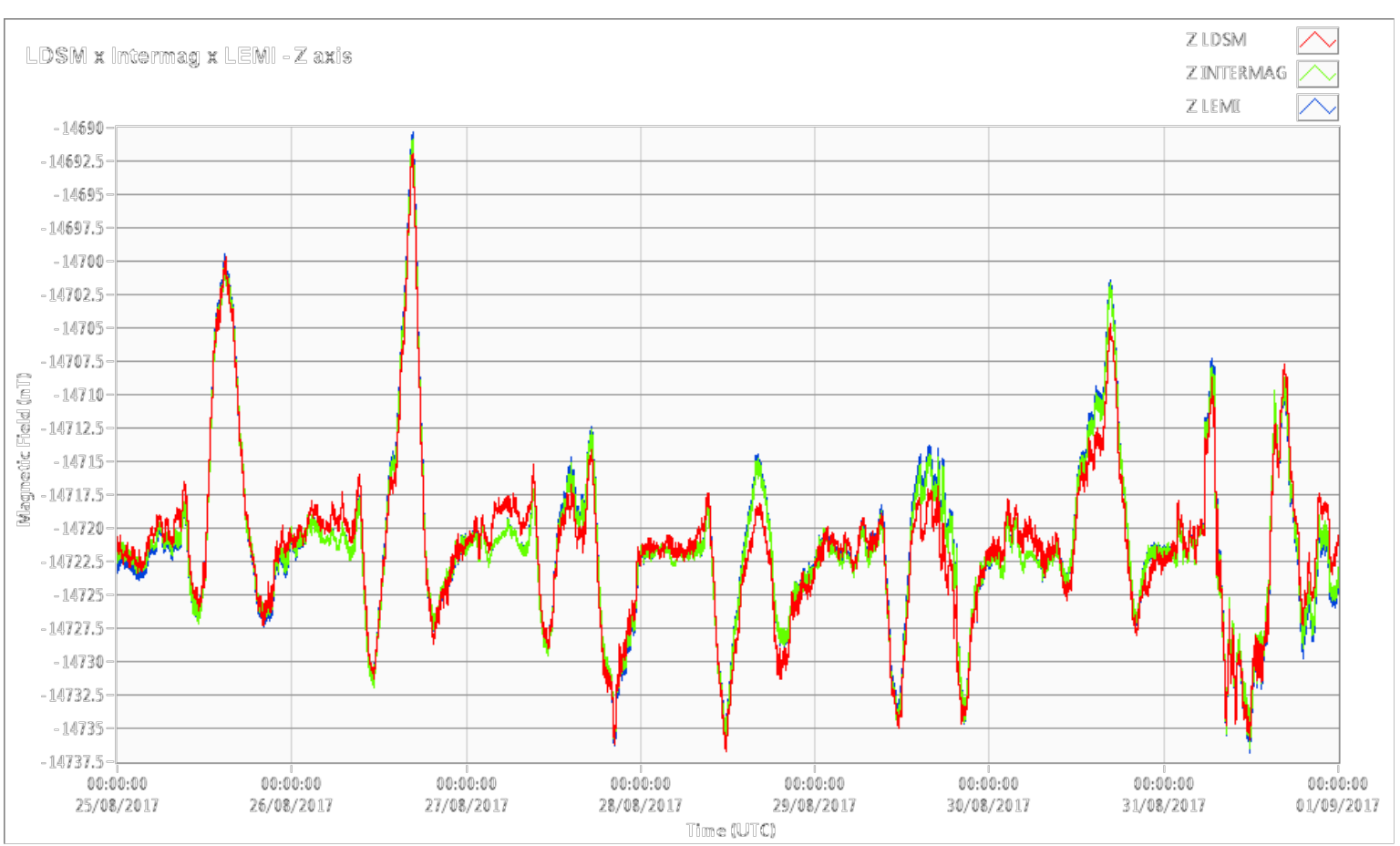

Figure 16. Long-term comparison between magnetometer developed by LDSM, LEMI-417 and INTERMAGNET Reference

\section{Discussion an Conclusions}

Through calibration tests performed with a new prototype magnetometer, specially built for this project, followed by a set of long-term comparative tests against different commercial magnetometers and a magnetic observatory reference, we were able to verify the successful application of the proposed method.

The tools developed in this work can be applied for calibration of existing instruments and for supporting the development of new magnetometers. Such a solution can be very attractive to other institutions in particular because it allows the dissemination of similar systems, as it contributes to the feasibility of adapting existing laboratories for the calibration of fluxgate magnetometers.

Acknowledgments: This work was developed with the support of the Observatório Nacional

Author Contributions: Conceptualization, Andre Wiermann; Data curation, Gustavo Cabral; Methodology, Gustavo Cabral; Software, Gustavo Cabral; Supervision, Andre Wiermann and Luiz C. C. Benyosef; Writing original draft, Gustavo Cabral; Writing - review and editing, Andre Wiermann.

Funding: This research received no external funding.

Conflicts of Interest: The authors declare no conflict of interest.

\section{References}

1. ACUÑA, M. H., 2002, “Space-based magnetometers”, Review of Scientific Instruments, v. 73, n. 11, pp. 3717. ISSN: 00346748. doi: 10.1063/1.1510570.

2. BENYOSEF, L. C. D. C., 2008, “Optimization of the Magnetic Properties of Materials for Fluxgate”, Materials Research, v. 11, n. 2, pp. 145-149.

3. BENYOSEF, L. C. C., WIERMANN, A., ROBERTO, J., et al., 2013, "MAGNETÔMETRO DE NÚCLEO SATURADO, DE ALTA RESOLUÇÃO, PARA APLICAÇÃO NAVAL SUBMERSA", pp. 80-91.

4. BRAUER, P., RISBO, T., MERAYO, J., et al., 2000, "Fluxgate sensor for the vector magnetometer onboard the 'Astrid-2' satellite", Sensors and Actuators A: Physical, v. 81, n. 1-3, pp. 184-188. ISSN: 09244247. doi: 10.1016/S0924-4247(99)00084-9. 
5. CERMAN, A., KUNA, A., RIPKA, P., et al., 2005, "Digitalization of highly precise fluxgate magnetometers", Sensors and Actuators, A: Physical, v. 121, n. 2, pp. 421-429. ISSN: 09244247. doi: 10.1016/j.sna.2005.03.053.

6. CSONTOS, A., HEGYMEGI, L., HEILIG, B., 2007, “Temperature Tests on Modern Magnetometers”, v. 99, n. 398.

7. ENGELTER, A., 1986, "A fluxgate magnetometer with a metallic glass core", IEEE transactions on magnetics, v. 22, n. 4 , pp. 299-300.

8. FORSTER, F., 1955, "A method for the measurement of DC field differences and its application to nondestructive testing", Nondestruct. Test, v. 13, pp. 31.

9. GERMAIN, C., 1963, "Bibliographical review of the methods of measuring magnetic fields", Nuclear Instruments and Methods, v. 21, pp. 17-46.

10. JANKOWSKI, J., SUCKSDORFF, C., 1996, “Guide for Magnetic Measurements and Observatory Practice”, p. 238.

11. KELLY, J. M., 1951, “Magnetic field measurements with peaking strips”, Review of Scientific Instruments, v. 22, n. 4, pp. 256-258.

12. KLIMOVICH, E., KOREPANOV, V., POSIO, P., et al., 2007, “Accredited Vector Magnetometer Calibration Facility", v. 43, pp. 59-76.

13. KOIKE, K., NAKAJIMA, S., SHIMIZU, Y., 1990, “On the Accuracy of Flux-Gate Magnetometers: Calibration Experiment", Memoirs of the Kakioka Magnetic Observatory, v. 24, n. 1, pp. 1-13.

14. MACHARET, D. G., PEREZ-IMAZ, H. I. A., REZECK, P. A. F., et al., 2016, “Autonomous Aeromagnetic Surveys Using a Fluxgate Magnetometer", Sensors, v. 16, pp. 1-19. doi: 10.3390/s16122169.

15. MATSUOKA, A., SHINOHARA, M., TANAKA, Y., et al., 2013, “Development of fluxgate magnetometers and applications Magnet Fields in the Space", An Introduction to Space Instrumentation, , n. 5, pp. 217-225.

16. MERAYO, J. M. G., PRIMDAHL, F., BRAUER, P., et al., 2001, “The orthogonalization of magnetic systems", Sensors and Actuators, A: Physical, v. 89, n. 3, pp. 185-196. ISSN: 09244247. doi: 10.1016/S0924-4247(00)00515$\mathrm{X}$.

17. NACIONAL, O., 2017. “Observatório Nacional desenvolve equipamento de alta tecnologia para operação naval submersa".Available online: <http://on.br/index.php/pt-br/ultimas-noticias/>.

18. PEDERSEN, E. B., PRIMDAHL, F., PETERSEN, J. R., et al., “A new type of fluxgate magnetometer for low magnetic fields", .

19. PIIL-HENRIKSEN, J., MERAYO, J. M., NIELSEN, O. V., et al., 1996, “Digital Detection and feedback Fluxgate Magnetometer", Measurement Science and Technology, v. 7, pp. 897-903. ISSN: 0957-0233. doi: 10.1088/0957-0233/7/6/006.

20. PRIMDAHL, F., 1979, “The fluxgate magnetometer”, Journal of Physics E: Scientific Instruments, v. 12, n. 4, pp. 241-253. ISSN: 0022-3735. doi: 10.1088/0022-3735/12/4/001. Available online: <http://stacks.iop.org/ 0022-3735/12/i=4/a=001?key=crossref.7a075a0b465d1017a2a358d6f5437b30>.

21. PRIMDAHL, F., HERNANDO, B., PETERSEN, J. R., et al., 1999, “Digital detection of the flux-gate sensor output signal", Measurement Science and Technology, v. 5, n. 4, pp. 359-362. ISSN: 0957-0233. doi: 10.1088/ 0957-0233/5/4/006.

22. PRIMDAHL, F., 1970, "Temperature Compensation of Fluxgate Magnetometers", IEEE Transactions on Magnetics, v. 6, n. 4, pp. 819-822. ISSN: 19410069. doi: 10.1109/TMAG.1970.1066971.

23. RIPKA, P., 2003, “Advances in fluxgate sensors", Sensors and Actuators, A: Physical, v. 106, n. 1-3, pp. 8-14. ISSN: 09244247. doi: 10.1016/S0924-4247(03)00094-3.

24. RIPKA, P., NAVRATIL, P., 1997, "Fluxgate sensor for magnetopneumometry", Sensors and Actuators A: Physical, v. 60, n. 1-3, pp. 76-79.

25. SON, D., 1989, “A new type of fluxgate magnetometer for low magnetic fields", Physica Scripta, v. 39, n. 4, pp. 535. Available online: <http:/ /stacks.iop.org/1402-4896/39/i=4/a=023>.

26. VACQUIER, V., SIMONS, R., HULL, A., 1947, “A magnetic airborne detector employing magnetically controlled gyroscopic stabilization", Review of Scientific Instruments, v. 18, n. 7, pp. 483-487.

27. VOPÁLENSKÝ, M., RIPKA, P., PLATIL, A., 2003, “Precise magnetic sensors", Sensors and Actuators, A: Physical, v. 106, n. 1-3, pp. 38-42. ISSN: 09244247. doi: 10.1016/S0924-4247(03)00129-8.

28. SCHULZ, L., HEINISCH, P., RICHTER, I, “Calibration of Off-the-Shelf Anisotropic Magnetoresistance Magnetometers", Sensors 2019, 19(8), 1850. 
485

486

487

488

$489-31$.

489

491

492

493

494

29. MURACCINI, M., MANGIA, A. L., LANNOCCA, M., AND CAPPELLO, A., “Magnetometer Calibration and Field Mapping through Thin Plate Splines", Sensors 2019, 19(2), 280.

30. WU, Z., AND WANG, W., "Magnetometer and gyroscope calibration method with level rotation", Sensors 2018, 18(3), 748.

1. YANG, D., YOU, Z., LI, B., DUAN, W., AND YUAN, B., “Complete tri-axis magnetometer calibration with a gyro auxiliary", Sensors 2017, 17(6), 1223.

32. RENAUDIN, V., AFZAL, M. H., AND LACHAPELLE, G., “Complete triaxis magnetometer calibration in the magnetic domain", Journal of Sensors 2010.

33. PETRUCHA, V., KASPAR, P., RIPKA, P., AND MERAYO, J. M., “Automated system for the calibration of magnetometers", Journal of Applied Physics 2009, 105(7), 07E704. 